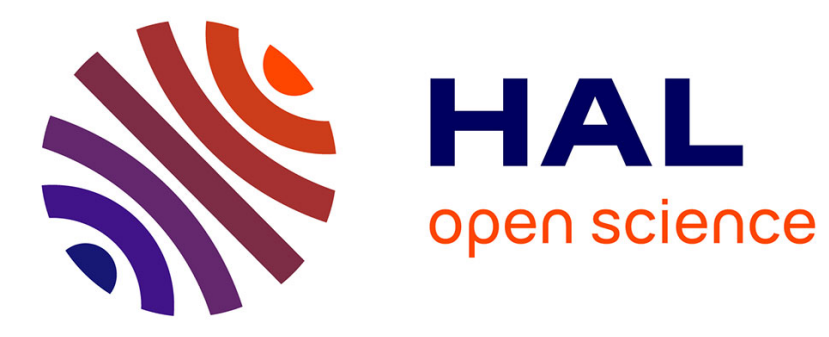

\title{
Convection behind the humidification of Titan's stratosphere
}

Pascal Rannou, Maélie Coutelier, Emmanuel D. Rivière, Sébastien Lebonnois, Michael M. Rey, L. Maltagliati

\section{- To cite this version:}

Pascal Rannou, Maélie Coutelier, Emmanuel D. Rivière, Sébastien Lebonnois, Michael M. Rey, et al.. Convection behind the humidification of Titan's stratosphere. The Astrophysical Journal, 2021, 922 (2), 10.3847/1538-4357/ac2904 . hal-03439334

\section{HAL Id: hal-03439334 https://hal.science/hal-03439334}

Submitted on 22 Nov 2021

HAL is a multi-disciplinary open access archive for the deposit and dissemination of scientific research documents, whether they are published or not. The documents may come from teaching and research institutions in France or abroad, or from public or private research centers.
L'archive ouverte pluridisciplinaire HAL, est destinée au dépôt et à la diffusion de documents scientifiques de niveau recherche, publiés ou non, émanant des établissements d'enseignement et de recherche français ou étrangers, des laboratoires publics ou privés. 
Convection behind the humidification of Titan's stratosphere

P. Rannou ${ }^{1}$, M. Coutelier ${ }^{1}$, E. Rivière ${ }^{1}$, S. Lebonnois ${ }^{2}$, M. Rey ${ }^{1}$ and L. Maltagliati ${ }^{3}$

${ }^{1}$ GSMA, Université de Reims Champagne-Ardenne, FRANCE

UMR 7331, CNRS,

Université de Reims Champagne-Ardenne,

Campus Sciences Exactes et Naturelles,

BP 1039, 51687 REIMS, FRANCE

Phone:(+33) 2269184 92,

e-mail: pascal.rannou@univ-reims.fr

${ }^{2}$ Laboratoire de Météorologie Dynamique, IPSL, Sorbonne Universté, PARIS, FRANCE

${ }^{3}$ Nature Astronomy, Springer Nature, 4 Crinan Street, N1 9XW LONDON, UNITED KINGDOM

CORRESPONDENCE TO:

P. Rannou

Groupe de Spectrométrie Moléculaire et Atmosphérique (UMR CNRS 7331)

UFR de Sciences Exactes et Naturelles, BP 1039

Université de Reims Champagne-Ardenne

51687 REIMS, FRANCE

Phone:(+33) 326918492

e-mail: pascal.rannou@univ-reims.fr 
1 Abstract : On Titan, methane is responsible for the complex prebiotic chemistry, the 2 global haze, most of the cloud cover and for the rainfalls which model the landscape. 3 Its sources are located in liquid reservoirs at and below the surface and it sink is 4 the photodissociation at high altitude. Titan's present and past climates strongly 5 depend on the connection between the surface sources and the atmosphere upper 6 layers. Despites its importance, very little information is available on this topic. In 7 this work, we re-analyze two solar occultations made by Cassini before the northern 8 spring equinox. We find a layer rich in methane at $165 \mathrm{~km}$ and at $70^{\circ} \mathrm{S}$ (mixing ratio $91.62 \pm 0.1 \%)$ and a dryer background stratosphere $(1.1-1.2 \%)$. In absence of local

production, this reveals an intrusion of methane transported into the stratosphere by convective circulation. On the other hand, methane transport through the tropopause at global scale appears quite inhibited. Leaking through the tropopause is an important bottleneck of Titan's methane cycle at all timescales. As such, it affects the long term evolution of Titan atmosphere and the exchange fluxes with the surface and subsurface reservoirs in a complex way. Global climate models (GCMs) accounting for cloud physics, thermodynamical feedbacks and convection are needed to understand the methane cycle, and specifically the humidificaton of the stratosphere, at the present time and its evolution under changing conditions at geological timescale.

1. Introduction : There are details about Titan's methane cycle which remain unknown. Methane main reservoirs are in lakes, seas and possibly in the subsurface (Mastrogiuseppe et al. (2014)). Efforts have been made to understand the main characteristics of the methane tropospheric cycle (Lora et al. (2015); Faulk et al. (2020)), that is the global fluxes in the troposphere and the surface ans subsurface sources and sinks. Radio-occultation measurements were used along with arguments involving circulation, observed cloud cover and wind measured by Huygens to state that, before the Northern Spring Equinox in 2009, the troposphere was more humid in the southern hemisphere than in the northern hemisphere (Tokano (2014)). This is a valuable, although quite loose, constraint. Using photometric observations collected in 2014, Lora and Ádámkovics (2017) found with certainty a very humid north polar region and, consistent with Tokano (2014) but not in a significant way, a methane humidity increasing from the north $\left(30-60^{\circ} \mathrm{N}\right)$ to the southern hemisphere $\left(30^{\circ} \mathrm{S}\right.$ to equator). However clear conclusions at global scale could not be drawn due to uncertainties in 
methane retrievals. Retrieving methane abundance is extremely difficult because optical properties of the haze and the tropospheric mist are not well defined. Observations of scattered light at the limb or in nadir viewing do not allow the disentanglement of methane abundance and particles without ambiguity. Thermal infrared emission observed with the Composite InfraRed Spectrometer (CIRS/Cassini) allows the retrieval of the methane mixing ratio, but with a low spatial resolution and only in the stratosphere. This is enough to describe latitudinal variations but not vertical profiles (Lellouch et al. (2014)). In the low and middle stratosphere, they generally found methane abundances lower than expected from Huygens (Niemann et al. (2010)) with strong variations and high values above the tropics and the polar regions, possibly connected to the circulation. With the Ultraviolet Spectrometer UVIS/Cassini, Yelle et al. (2021) also found a low methane mixing ratio, around $1 \%$ at $950 \mathrm{~km}$, around the homopause.

We re-analyzed two occultations made by the Visual Infrared Mapping Spectrometer (VIMS/Cassini) during the flybys T10 in January 2006 and T53 in April 2009, previously published and analyzed (Maltagliati et al. (2015)). These observations are performed before the equinoctial turnover in 2009, while the large scale circulation has remained steady for a Titan's season and a half $(\simeq 11$ terrestrial years) (Rannou et al. (2004); Lebonnois et al. (2012)). We used the methane linelist published by Rey et al. (2018) which significantly improves the quality of the retrievals. Details about the model, the results and comparisons with the previous analysis are discussed in the Supplementary Information. Occultations probe extinction along tangential lines of sight at the limb and they easily allow to seperate methane and haze extinction. In the following, we discuss our retrieval for the methane mixing ratio.

2. Description of the method : To proceed to the analysis, we calculate the haze and the gas properties in Titan's atmosphere following Rannou et al. (2018) and Coutelier et al. (2021). Since we deal with occultation data, only extinction properties are needed. Then, we built a simple model for computing tangential transmissions through an atmosphere, as observed with the occultation mode. The vertical grid of the model is adjusted to the number of data $N_{D}$. We then define as much as layers than data points. We set the haze properties and the methane abundance at the lower boundary (or impact factor) $z_{i}$ of each layers, with $i=1$ to $N_{D}$, as free parameters. The forward model is able to produce transmissions $\boldsymbol{T}_{m}\left(z_{i}\right)$ at the impact factors $z_{i}$ as a function the haze properties (extinction $\boldsymbol{k}_{H}\left(z_{i}\right)$ and spectral slope $\boldsymbol{\alpha}_{H}\left(z_{i}\right)$ ) and the gas methane mixing ratio $\boldsymbol{X}_{C H 4}\left(z_{i}\right)$, 
diretly linked to the gas extinction $\left(\boldsymbol{k}_{\mathrm{CH}}\left(z_{i}\right)\right)$. The model transmissions $\boldsymbol{T}_{m}\left(z_{i}\right)$ are compared with the observed transmissions $\boldsymbol{T}_{o}\left(z_{i}\right)$. To produce the retrieval, we use a bayesian method along with a diffusive process to stabilize the algorithm. This is very suitable for this kind of triangular problems, that is when the transmission at a given level only depends on the atmosphere properties above this level. It is also convenient because only jacobians of the model are needed and the retrieval is performed with the iterative resolution of a matricial equation (See Appendix A and B for details). This method is very close to the one used by Maltagliati et al. (2015) and only the better methane linelist provided by Rey et al. (2018) brings a decisive improvement in our work.

3. Constrasted stratospheric methane profiles : The Figure 1 shows the retrieved vertical profiles for the two observations and the corresponding fits of the data. The methane profile at $70^{\circ} \mathrm{S}(T 10)$ displays an enriched layer, with a mixing ratio up to $1.62 \pm 0.10 \%$ at $165 \mathrm{~km}$, isolated in a relatively dry background atmosphere. Above this layer, the methane mixing ratio is quite constant with a value $\simeq 1.05 \pm 0.10 \%$. Below, it drops to $\simeq 0.70 \pm 0.20 \%$ around $125 \mathrm{~km}$. With the T53 observation, at $1^{\circ} \mathrm{N}$, we find stratospheric values oscillating around $1.15 \%$ with error bars and amplitude both around $0.05 \%$ above $200 \mathrm{~km}$ and with a wavelength of $35_{-3.0}^{+4.5} \mathrm{~km}$. Huygens found, near the equator, that methane decreases from 5\% to 1.5\% (Niemann et al. (2005); Niemann et al. (2010)) in the troposphere due to condensation. We then find that it further decreases to reach values around 1.0 to $1.15 \%$ in the mid-stratosphere at low latitudes instead of being constant as generally assumed. Our results are consistent with the effective values retrieved with CIRS in broad altitude ranges at $125 \pm 50 \mathrm{~km}$ and $225 \pm 50 \mathrm{~km}$. However, our vertical resolutions allow us to be much more assertive and lift ambiguities about the mechanisms behind this methane distribution.

The Figure 2 shows our results for the methane mixing ratios along with other values retrieved in the stratosphere and values evaluated at the cold trap (where the mixing ratio of air is capped due to the limitation by the saturation vapor pressure) from temperature measurements. Temperatures are stable with time in the low stratosphere (1 to 10 mbar) between 2005 and 2009 (Achterberg et al. (2011)) and are expected to be even more stable at higher pressure (Lebonnois et al. (2012)). We then consider temperatures gathered from data collected between 2005 and 2009, as a consistent set which depicts a steady state (Lellouch et al. (2014)). On the other hand, post-equinoctial changes are extremely rapid, even at the scale of a terrestrial year (e.g., Lebonnois et al. (2012); West et al. (2018); Teanby et al. (2019); Seignovert et al. (2021)). Thus, we ignore in our discussion data taken 
after equinox.

At first glance, the methane mixing ratio at $125 \pm 50 \mathrm{~km}$ found by Lellouch et al. (2014) are highly variable with latitude. They appears not related to the latitudinal temperatures at the cold trap and the corresponding saturation mixing ratios, indicating a disconnection between the two fields (Figure 2). Near equator (T53), the mixing ratio $1.25 \pm 0.25 \%$ around $150 \mathrm{~km}$ is also lower, yet consistent, compared to the value found by the GC-MS (Gas Chromatograph and Mass Spectrometer) at the cold trap. The value $1.15 \pm 0.05 \%$ around $250 \mathrm{~km}$ is much lower and not consistent with the value found at cold trap or in the low stratosphere with the GC-MS. At $70^{\circ} \mathrm{S}$ (T10), the methane mixing ratio of $1.62 \pm 0.10 \%$ in the enriched layer at $165 \mathrm{~km}$ is much larger than the maximum value allowed by the cold trap temperature at $70^{\circ} \mathrm{S}$, that is $\simeq 1.05 \pm 0.19 \%$. On the other hand, this value is comparable to values allowed by the cold trap between tropics. There is no local source of methane in the atmosphere and we do not expect that an eventual wave system could concentrate methane up to mixing ratios exceeding the background value by $50 \%$. Such a layer is then a clear signature of an intrusion coming from a moist environment at another latitude. These results undermine the simple idea of a gaseous vertical flux through the tropopause at global scale, locally limited by the saturation vapor pressure.

4. Methane profiles decyphered with Titan's circulation : To understand our results, we first discuss Titan's circulation with the results of a GCM (Rannou et al. (2006)). Although this is a 2 dimensional (latitude-pressure) model, it accounts for the haze seasonal transport at global scale up to the mesosphere, the cloud microphysics and the radiative feedbacks. It predicts well the thick winter haze polarhood and the haze scavenging by clouds in the troposphere. The corresponding opacity fields are important drivers of the circulation through the control of the solar flux at the surface and the cooling to space during the polar night (e.g., Rannou et al. (2004)). To date, no existing 3-D model accounts for all these couplings although they have major effects on the troposphere and low stratosphere circulation.

GCMs long predicted two opposite circulation cells below the stratosphere temperature inversion (Rannou et al. (2006)) and a large thermally direct cell in the stratosphere with rapid postequinoctial turnover (e.g., Rannou et al. (2004); Lebonnois et al. (2012); Larson et al. (2014); Lora et al. (2015)) (Figure 3). Before the Northern Spring Equinox in 2009, at the time of T10 
and $T 53$, both troposphere cells have a lower branch near surface which converge around $30-40^{\circ} \mathrm{S}$

to give an ascending branch emerging from the troposphere (e.g., Rannou et al. (2006)). This is equivalent to the tropospheric Hadley cells and the Inter-Tropical Convergence Zone (ITCZ) on Earth, except that cells extend up to the poles because of Titan's slow rotation speed. Observation of the tropical clouds on Titan reveals the actual location of the Tropical Convergence Zone (here after TCZ) around $\simeq 45 \pm 15^{\circ} \mathrm{S}$ (Rodriguez et al. (2011); Turtle et al. (2018)). The prediction of clouds related to the Hadley-like cells in the coupled GCM (Rannou et al. (2006)) at the location where they are actually observed validates this model for our discussion. However, it should be reminded that its results are also zonally and time averaged. The sporadic nature of the cloud events, the short term fluctuations and longitude variabilities of the circulation are then erased.

At the TCZ, the ascending winds splits in two branches in the low stratosphere to close the Hadleylike cells. One cell drives air from the southern tropical tropopause to the south polar region up to around $150-200 \mathrm{~km}$ and sink near the south pole. The other cell is forced to return equatorward almost horizontally at around $70 \mathrm{~km}$ altitude (upper branch) because the strong stratosphere temperature inversion, with high hydrostatic stability, prevents air from moving upward between tropics. This circulation leaves a strong dynamical signature with the zonal wind dropping due to the conservation of angular momentum. This dynamical signature is also observed at $70 \mathrm{~km}$ in reality, with zonal winds of $4 \mathrm{~m} / \mathrm{s}$ in a thin layer of about $10 \mathrm{~km}$ thick while layers above and below have zonal winds larger that $40-50 \mathrm{~m} / \mathrm{s}$ (Bird et al. (2005)). This specific layer makes a sharp transition between the Hadley-like cell and the stratosphere cell (Rannou et al. (2006); Lebonnois et al. (2012); Larson et al. (2014)).

Methane mole fractions superimposed to the circulation pattern (Figure 3) show that the methanerich layer observed during $T 10$ is directly linked to an ascending branch which comes from the moist troposphere at the TCZ. It can convey methane to the south polar region up to about 160 $\mathrm{km}$, above the cold and dry polar tropopause and explains the intrusion layer. For T53, we know that in the inter-tropical region, the transition in methane abundance from $1.5 \%$ to $1.15 \%$ (and $\simeq 1 \%$ in Lellouch et al. (2014)) takes place at the interface between the Hadley-like cell and the thermally direct stratosphere cell. The sharp drop in the zonal wind at the same altitude (Bird et al. (2005)) associated to horizontal winds and to a strong temperature inversion indicates inhibited vertical exchanges. It marks the transition between a moist troposphere and low stratosphere, on one side, 
and the dryer background stratospheric air fed in methane through intrusions at some latitudes and not globally, on the other side. Clearly, the equatorial region is not one of these intrusion zones.

5. Humidification by convection on Titan and on Earth : The dynamical origin of the stratospheric methane distribution is obvious in observations, however GCMs accounting for non convective cloud microphysics and transport of species are not able to produce such distribution patterns (e.g., Rannou et al. (2006)). Instead, they predict a constant methane mixing ratio in the stratosphere and the mesosphere that directly depends on the mixing ratio at the tropopause. This discrepancy underlines the role of cloud convection in transfering methane upward above the cold trap and into the stratosphere. Between 2004 and 2010, clouds are frequently observed at the TCZ (Rodriguez et al. (2011); Turtle et al. (2018)) and briefly near equator during the equinoctial transition (Turtle et al. (2011)). A photometric analysis shows that the core of these cloud systems can reach the high troposphere and the tropopause (Griffith et al. (2005)). Mesoscale cloud models also show that with a surface humidity larger than $50 \%$ or $65 \%$, convective clouds triggered by a perturbation in a moving air mass (Barth (2010)) or in a static atmosphere easily reach high altitudes (Barth and Rafkin $(2007,2010)$ ). Large scale ascending motions in the Hadley-like cells could trigger and facilitate convection. Convective clouds also inject methane ice particles, rather than vapor, without limitation due to the saturation vapor pressure at the cold trap. This would further enhance contrasts in the stratospheric methane distribution.

On Earth, by comparison, the entry of water vapour in the stratosphere between tropics and at midlatitudes is due to a slow gaseous advection, capped by cloud condensation, and also to overshooting of convective ice clouds in the lower stratosphere (e.g., Liu et al. (2010)). Convective overshoots marginally account for several percents of the total flux of water, less than $18 \%$ according to the largest estimates (e.g., Ueyama et al. (2018); Dauhut et al. (2015)). As a direct consequence, average water abundance in the tropical stratosphere is relatively homogenous and well related to the tropopause temperature. Only oxidation of methane further produces inhomogeneities in the water stratospheric abundance. The Earth's case enlighten Titan's case ; on Earth, transport dominated by slow advection yields a quite uniform water mixing ratio in the stratosphere. On the contrary, on Titan, a substantial transfer by convection and an inhibited slow convection explains the sharp distribution in the stratosphere and a low background mixing ratio. In both atmospheric systems, slow advection and strong convective transports at the tropopause exist, but they have not 
the same relative strenghs. This difference in transports through the tropopause causes different relationship between the tropospheric and stratospheric mixing ratio.

6. Consequence on short and long term climate : After Huygens observations, the stratospheric methane abundance $1.4 \%$ is often used as a fiducial value to study other components of Titan (haze, clouds, temperature, other species, surface reflectivity, thermal equilibrium). This is incorrect in two ways. First, the methane stratospheric abundance is around 1 to $1.1 \%$ in average rather than 1.4\%. Secondly, its value is highly variable with space and probably with time. A simple test using the model of Coutelier et al. (2021) shows that a low methane abundance affects the retrieved haze opacity in stratosphere by $\simeq-4 \%$ and the tropospheric mist opacity by $\simeq+7 \%$. Although apparently moderate, these changes are about twice the accumulated errors produced by the instrumental uncertainties and the model bias on the retrieved values. Such a low methane abundance also sets new conditions for the amount and the distribution of methane in the upper atmosphere (Yelle et al. (2021)), with probable consequences on the escape rates and on the composition of aerosols and minor species.

More generally, humidification by convective entry and inhibition of slow vertical transfer also sets new conditions for the evaluation of the long term climates of Titan (Lunine and Atreya (2008); Hayes et al. (2018)). This way to regulate the stratosphere humidification strongly depends on the thermodynamical conditions behind cloud formation and convection, known to have onset and cut-off thresholds (e.g., Barth and Rafkin (2007, 2010),Barth (2010)). Other climatic conditions, in the past or in the future, may radically change the way methane is transported upward due to convective motions. This would dim or enhance the flux of methane available for photochemistry much more and in a more complex way than anticipated with simple models based on slow advective transport (e.g., Lorenz et al. (1999)). Of course, it would also change our perception of Titan history in shaking the exchange timescales and fluxes between the different methane reservoirs in Titan. Future progress in understanding the methane cycle on Titan will strongly rely on efficient and complete global climate models, associated to mesoscale models, able to treat the cloud microphysics and the moist convection process in detailed aspects. 


\section{References}

Achterberg, R., Gierasch, P., Conrath, B., Michael Flasar, F., and Nixon, C. (2011). Temporal variations of titan's middle-atmospheric temperatures from 2004 to 2009 observed by cassini/cirs. Icarus, 211(1):686-698.

Barth, E. and Rafkin, S. (2007). Trams: A new dynamic cloud model for titan's methane clouds. Geophysical Research Letters, 34(3).

Barth, E. and Rafkin, S. (2010). Convective cloud heights as a diagnostic for methane environment on titan. Icarus, 206(2):467-484.

Barth, E. L. (2010). Cloud formation along mountain ridges on titan. Planetary and Space Science, 58(13):1740 - 1747. Surfaces and atmospheres of the outer planets, their satellites and ring systems: Part VI.

Bellucci, A., Sicardy, B., Drossart, P., Rannou, P., Nicholson, P. D., Hedman, M., Baines, K. H., and Burrati, B. (2009). Titan solar occultation observed by Cassini/VIMS: Gas absorption and constraints on aerosol composition. Icarus, 201:198-216.

Bézard, B. (2014). The methane mole fraction in Titan's stratosphere from DISR measurements during the Huygens probe's descent. Icarus, 242:64-73.

Bird, M. K., Allison, M., Asmar, S. W., Atkinson, D. H., Avruch, I. M., Dutta-Roy, R., Dzierma, Y., Edenhofer, P., Folkner, W. M., Gurvits, L. I., Johnston, D. V., Plettemeier, D., Pogrebenko, S. V., Preston, R. A., and Tyler, G. L. (2005). The vertical profile of winds on titan. Nature, 438(7069):800-802.

Coutelier, M., Cordier, D., Seignovert, B., Rannou, P., Le Gall, A., Cours, T., Maltagliati, L., and Rodriguez, S. (2021). Distribution and intensity of water ice signature in south xanadu and tui regio. Icarus, 364:114464.

Dauhut, T., Chaboureau, J.-P., Escobar, J., and Mascart, P. (2015). Large-eddy simulations of hector the convector making the stratosphere wetter. Atmospheric Science Letters, 16(2):135140. 
Faulk, S., Lora, J., Mitchell, J., and Milly, P. (2020). Titan's climate patterns and surface methane distribution due to the coupling of land hydrology and atmosphere. Nature Astronomy, 4(4):390398.

Fray, N. and Schmitt, B. (2009). Sublimation of ices of astrophysical interest: A bibliographic review. Planet. Space Sci., 57:2053-2080.

Fulchignoni, M., Ferri, F., Angrilli, F., Ball, A. J., Bar-Nun, A., Barucci, M. A., Bettanini, C., Bianchini, G., Borucki, W., Colombatti, G., Coradini, M., Coustenis, A., Debei, S., Falkner, P., Fanti, G., Flamini, E., Gaborit, V., Grard, R., Hamelin, M., Harri, A. M., Hathi, B., Jernej, I., Leese, M. R., Lehto, A., Lion Stoppato, P. F., López-Moreno, J. J., Mäkinen, T., McDonnell, J. A. M., McKay, C. P., Molina-Cuberos, G., Neubauer, F. M., Pirronello, V., Rodrigo, R., Saggin, B., Schwingenschuh, K., Seiff, A., Simões, F., Svedhem, H., Tokano, T., Towner, M. C., Trautner, R., Withers, P., and Zarnecki, J. C. (2005). In situ measurements of the physical characteristics of Titan's environment. Nature, 438:785-791.

Goody, R., West, R., Chen, L., and Crisp, D. (1989). The correlated-k method for radiation calculations in nonhomogeneous atmospheres. J. Quant. Spectrosc. Radiat. Transf., 42:539-550.

Gordon, I., Rothman, L., Hill, C., Kochanov, R., Tan, Y., Bernath, P., Birk, M., Boudon, V., Campargue, A., Chance, K., Drouin, B., Flaud, J.-M., Gamache, R., Hodges, J., Jacquemart, D., Perevalov, V., Perrin, A., Shine, K., Smith, M.-A., Tennyson, J., Toon, G., Tran, H., Tyuterev, V., Barbe, A., Császár, A., Devi, V., Furtenbacher, T., Harrison, J., Hartmann, J.-M., Jolly, A., Johnson, T., Karman, T., Kleiner, I., Kyuberis, A., Loos, J., Lyulin, O., Massie, S., Mikhailenko, S., Moazzen-Ahmadi, N., Mller, H., Naumenko, O., Nikitin, A., Polyansky, O., Rey, M., Rotger, M., Sharpe, S., Sung, K., Starikova, E., Tashkun, S., Auwera, J. V., Wagner, G., Wilzewski, J., Wciso, P., Yu, S., and Zak, E. (2017). The hitran2016 molecular spectroscopic database. Journal of Quantitative Spectroscopy and Radiative Transfer, 203:3 - 69. HITRAN2016 Special Issue.

Griffith, C. A., Penteado, P., Baines, K., Drossart, P., Barnes, J., Bellucci, G., Bibring, J., Brown, R., Buratti, B., Capaccioni, F., Cerroni, P., Clark, R., Combes, M., Coradini, A., Cruikshank, D., Formisano, V., Jaumann, R., Langevin, Y., Matson, D., McCord, T., Mennella, V., Nelson, R., Nicholson, P., Sicardy, B., Sotin, C., Soderblom, L. A., and Kursinski, R. (2005). The Evolution of Titan's Mid-Latitude Clouds. Science, 310:474-477. 
Hayes, A., Lorenz, R., and Lunine, J. (2018). A post-cassini view of titan's methane-based hydrologic cycle. Nature Geoscience, 11(5):306-313.

Koskinen, T. T., Yelle, R. V., Snowden, D. S., Lavvas, P., Sandel, B. R., Capalbo, F. J., Benilan, Y., and West, R. A. (2011). The mesosphere and lower thermosphere of Titan revealed by Cassini/UVIS stellar occultations. Icarus, 216(2):507-534.

Larson, E. J., Toon, O. B., and Friedson, A. J. (2014). Simulating titan's aerosols in a three dimensional general circulation model. Icarus, 243:400 - 419 .

Lebonnois, S., Burgalat, J., Rannou, P., and Charnay, B. (2012). Titan global climate model: A new 3-dimensional version of the IPSL Titan GCM. Icarus, 218(1):707-722.

Lellouch, E., Bézard, B., Flasar, F. M., Vinatier, S., Achterberg, R., Nixon, C. A., Bjoraker, G. L., and Gorius, N. (2014). The distribution of methane in Titan's stratosphere from Cassini/CIRS observations. Icarus, 231:323-337.

Liu, X. M., Rivière, E. D., Marécal, V., Durry, G., Hamdouni, A., Arteta, J., and Khaykin, S. (2010). Stratospheric water vapour budget and convection overshooting the tropopause: modelling study from scout-amma. Atmospheric Chemistry and Physics, 10(17):8267-8286.

Lora, J. M. and Ádámkovics, M. (2017). The near-surface methane humidity on titan. Icarus, $286: 270-279$.

Lora, J. M., Lunine, J. I., and Russell, J. L. (2015). Gcm simulations of titan's middle and lower atmosphere and comparison to observations. Icarus, 250:516 - 528.

Lorenz, R. D., McKay, C. P., and Lunine, J. I. (1999). Analytic investigation of climate stability on titan: sensitivity to volatile inventory. Planetary and Space Science, 47(12):1503 - 1515.

Lunine, J. and Atreya, S. (2008). The methane cycle on titan. Nature Geoscience, 1(3):159-164.

Maltagliati, L., Bézard, B., Vinatier, S., Hedman, M. M., Lellouch, E., Nicholson, P. D., Sotin, C., de Kok, R. J., and Sicardy, B. (2015). Titan's atmosphere as observed by Cassini/VIMS solar occultations: $\mathrm{CH}_{4}, \mathrm{CO}$ and evidence for $\mathrm{C}_{2} \mathrm{H}_{6}$ absorption. Icarus, 248:1-24. 
Mastrogiuseppe, M., Poggiali, V., Hayes, A., Lorenz, R., Lunine, J., Picardi, G., Seu, R., Flamini, E., Mitri, G., Notarnicola, C., Paillou, P., and Zebker, H. (2014). The bathymetry of a titan sea. Geophysical Research Letters, 41(5):1432-1437.

Niemann, H., Atreya, S., Bauer, S., Carignan, G., Demick, J., Frost, R., Gautier, D., Haberman, J., Harpold, D., Hunten, D., Israel, G., Lunine, J., Kasprzak, W., Owen, T., Paulkovich, M., Raulin, F., Raaen, E., and Way, S. (2005). The abundances of constituents of titan's atmosphere from the gcms instrument on the huygens probe. Nature, 438(7069):779-784.

Niemann, H. B., Atreya, S. K., Demick, J. E., Gautier, D., Haberman, J. A., Harpold, D. N., Kasprzak, W. T., Lunine, J. I., Owen, T. C., and Raulin, F. (2010). Composition of Titan's lower atmosphere and simple surface volatiles as measured by the Cassini-Huygens probe gas chromatograph mass spectrometer experiment. Journal of Geophysical Research (Planets), 115(E14):E12006.

Quémerais, E., Bertaux, J.-L., Korablev, O., Dimarellis, E., Cot, C., Sandel, B. R., and Fussen, D. (2006). Stellar occultations observed by SPICAM on Mars Express. Journal of Geophysical Research (Planets), 111(E9):E09S04.

Rannou, P., Cabane, M., Botet, R., and Chassefière, E. (1997). A new interpretation of scattered light measurements at Titan's limb. Journ. Geoph. Res., 102:10997-11014.

Rannou, P., Hourdin, F., McKay, C., and Luz, D. (2004). A coupled dynamics-microphysics model of titan's atmosphere. Icarus, 170(2):443 - 462 .

Rannou, P., Montmessin, F., Hourdin, F., and Lebonnois, S. (2006). The latitudinal distribution of clouds on titan. Science, 311(5758):201-205.

Rannou, P., Seignovert, B., Le Mouélic, S., Maltagliati, L., Rey, M., and Sotin, C. (2018). Transparency of $2 \mu \mathrm{m}$ window of titan's atmosphere. Planetary and Space Science, 151:109 - 124.

Rey, M., Nikitin, A., Bézard, B., Rannou, P., Coustenis, A., and Tyuterev, V. (2018). New accurate theoretical line lists of $12 \mathrm{ch} 4$ and $13 \mathrm{ch} 4$ in the $0-13400 \mathrm{~cm}-1$ range: Application to the modeling of methane absorption in titan's atmosphere. Icarus, 303:114 - 130.

Rodgers, C. D. (2000). Inverse methods for atmospheric sounding : theory and practice. World Scientific Publishing. 
Rodriguez, S., Le Moulic, S., Rannou, P., Sotin, C., Brown, R., Barnes, J., Griffith, C., Burgalat, J., Baines, K., Buratti, B., Clark, R., and Nicholson, P. (2011). Titan's cloud seasonal activity from winter to spring with cassini/vims. Icarus, 216(1):89-110.

Schinder, P., Flasar, F., Marouf, E., French, R., McGhee, C., Kliore, A., Rappaport, N., Barbinis, E., Fleischman, D., and Anabtawi, A. (2012). The structure of titan's atmosphere from cassini radio occultations: Occultations from the prime and equinox missions. Icarus, 221(2):1020-1031.

Schinder, P. J., Flasar, F. M., Marouf, E. A., French, R. G., Anabtawi, A., Barbinis, E., Fleischman, D., and Achterberg, R. K. (2020). The structure of titan's atmosphere from cassini radio occultations: One- and two-way occultations. Icarus, 345:113720.

Schinder, P. J., Flasar, F. M., Marouf, E. A., French, R. G., McGhee, C. A., Kliore, A. J., Rappaport, N. J., Barbinis, E., Fleischman, D., and Anabtawi, A. (2011). The structure of titan's atmosphere from cassini radio occultations. Icarus, 215(2):460-474.

Seignovert, B., Rannou, P., West, R. A., and Vinatier, S. (2021). Haze seasonal variations of titan's upper atmosphere during the cassini mission. The Astrophysical Journal, 907(1):36.

Teanby, N., Sylvestre, M., Sharkey, J., Nixon, C., Vinatier, S., and Irwin, P. (2019). Seasonal evolution of titan's stratosphere during the cassini mission. Geophysical Research Letters, 46(6):30793089.

Tokano, T. (2014). Non-uniform global methane distribution in titan's troposphere evidenced by cassini radio occultations. Icarus, 231:1 - 12 .

Tokano, T., McKay, C., Neubauer, F., Atreya, S., Ferri, F., Fulchignoni, M., and Niemann, H. (2006). Methane drizzle on titan. Nature, 442(7101):432-435.

Turtle, E., Perry, J., Hayes, A., Lorenz, R., Barnes, J., McEwen, A., West, R., Del Genio, A., Barbara, J., Lunine, J., Schaller, E., Ray, T., Lopes, R., and Stofan, E. (2011). Rapid and extensive surface changes near titan's equator: Evidence of april showers. Science, 331(6023):1414-1417.

Turtle, E. P., Perry, J. E., Barbara, J. M., Del Genio, A. D., Rodriguez, S., Le Moulic, S., Sotin, C., Lora, J. M., Faulk, S., Corlies, P., Kelland, J., MacKenzie, S. M., West, R. A., McEwen, A. S., Lunine, J. I., Pitesky, J., Ray, T. L., and Roy, M. (2018). Titan's meteorology over the cassini 
mission: Evidence for extensive subsurface methane reservoirs. Geophysical Research Letters, 45(11):5320-5328.

Ueyama, R., Jensen, E. J., and Pfister, L. (2018). Convective influence on the humidity and clouds in the tropical tropopause layer during boreal summer. Journal of Geophysical Research: Atmospheres, 123(14):7576-7593.

West, R. A., Balloch, J., Dumont, P., Lavvas, P., Lorenz, R., Rannou, P., Ray, T., and Turtle, E. P. (2018). The evolution of titan's detached haze layer near equinox in 2009. Geophysical Research Letters, 38(6).

Yelle, R. V., Koskinen, T., and Palmer, M. (2021). Titan occultations of orion's belt observed with cassini/uvis. Icarus, page 114587. 
Appendix A The retrieval model: Occultations probe extinction along lines of sight at the limb of the planet and are not sensitive to multiple scattering due to lower layers or to surface reflectivity. This kind of data easily allows to separate methane and haze extinction since they extinct light in very different ways. To match data, we divide atmosphere according to the vertical sampling of observations and we compute transmitted flux accounting haze and gas extinction in each layer. We use data between $0.8 \mu \mathrm{m}$ and $2.0 \mu \mathrm{m}$, where the signal is largely dominated by haze and methane extinction. Other methane bands, at $2.3 \mu \mathrm{m}$ and $3.4 \mu \mathrm{m}$, are mixed with other undefined absorptions. This has been long remarked for the $3.4 \mu \mathrm{m}$ fundamental band (e.g., Maltagliati et al. (2015)) but, in this work, we also find a prominent unknown absorption that we discuss later. Methane absorption is given by Rey et al. (2018) linelist and is treated with the correlated-k method (Goody et al. (1989)) with 16 terms. Other gas properties are set with linelists published in the Hitran database (Gordon et al. (2017)). The mixing ratio $X_{C H 4}$ is the only free parameter relative to methane given for each layer. Haze extinction is computed with a model of scattering and absorption by fractal aggregates (Rannou et al. (1997)) and the main characteristics of haze could be summarized as a vertical profile of extinction coefficient $k_{H}$ at a reference wavelength, $\lambda_{0}$, and the spectral slope $\alpha H=\partial k_{H}(\lambda) / \partial \lambda$.

Appendix B Method related to the retrieval procedure: To produce our analysis, we developed a retrieval model following the technique of the bayesian inference (Rodgers (2000)). Using the Bayes theorem, this technique allows for retrieving a parameter set $\boldsymbol{x}$ along with an estimated error $\sigma_{\boldsymbol{x}}$ from a set of observation $\boldsymbol{y}$ with observation errors $\boldsymbol{\sigma}_{\boldsymbol{y}}$. Each set is represented by a vector. The strengh of this approach essentially relies on the fact that we only need a knowledge about the direct relationship between $\boldsymbol{y}$ and $\boldsymbol{x}$. This relation is formally given by $\boldsymbol{y}=F(\boldsymbol{x})$, where $F$ stands, in our case, for the radiative transfer model that the links observed transmissions $(\boldsymbol{y})$ and the atmosphere parameters $(\boldsymbol{x})$ such as the haze extinction or gas mixing ratios.

To proceed, the function $F$ has to be linearized around a given solution $\boldsymbol{y}\left(\boldsymbol{x}_{\mathbf{0}}\right)=\boldsymbol{y}_{\mathbf{0}}+\partial F(\boldsymbol{x}) / \partial \boldsymbol{x} \times$ $\left(\boldsymbol{x}-\boldsymbol{x}_{\mathbf{0}}\right)$, and where $\boldsymbol{y}_{\mathbf{0}}=F\left(\boldsymbol{x}_{\mathbf{0}}\right)$. In this way, the set of partial derivatives $\partial F\left(\boldsymbol{x}_{\mathbf{0}}\right) / \partial \boldsymbol{x}$ yields a matrix $K$ and the estimation for the best value of $\overline{\boldsymbol{x}}$ can be written as (Rodgers (2000)) : 


$$
\bar{x}=x_{0}+\left(K^{T} S_{\epsilon}^{-1} K\right)^{-1} K^{T} S_{\epsilon}^{-1}\left(y-y\left(x_{0}\right)\right)
$$

where $\boldsymbol{S}_{\boldsymbol{\epsilon}}$ is the diagonal error matrix $\left(\boldsymbol{S}_{\boldsymbol{\epsilon},(\boldsymbol{i} \boldsymbol{j})}=\delta_{i, j} / \sigma_{\boldsymbol{y}_{\boldsymbol{i}}}^{2}\right), \boldsymbol{S}=\left(\boldsymbol{K}^{\boldsymbol{T}} \boldsymbol{S}_{\boldsymbol{\epsilon}}^{-\mathbf{1}} \boldsymbol{K}\right)^{-\mathbf{1}}$ is the covariance matrix and $\boldsymbol{y}$ is the observation vector. If $\boldsymbol{x}_{\mathbf{0}}$ is far from the best solution, the first estimated value $\overline{\boldsymbol{x}}$ will not be the best possible solution but will be better than $\boldsymbol{x}_{\mathbf{0}}$. So we can start an iterative process to converge toward a solution which will be the most likely solution. In general, 20 to 40 iterations are needed to obtain an acceptable solution. It depends on the size of the vector $\boldsymbol{x}$. Notably, although we start with an initial guess for $\boldsymbol{x}_{\mathbf{0}}$, we do not include any a priori value in the retrieval matrix. The error on the retrieved parameter $\boldsymbol{x}$ are given by the square root of the diagonal terms in the covariance matrix $\sigma_{x i}=\sqrt{S_{i i}}$

In order to avoid instabilities in the converged solution, we used a Tikhonov regularization. This consists in adding a process analogous to a diffusion in the $\mathbf{x}$-space to attenuate spurious oscillations. It takes the form of a matrix, $\mathbf{H}$, inserted in the covariance matrix as:

$$
\boldsymbol{S}^{\dagger}=\left(\boldsymbol{K}^{\boldsymbol{T}} \boldsymbol{S}_{\boldsymbol{\epsilon}}^{-\mathbf{1}} \boldsymbol{K}+\beta \boldsymbol{H}\right)^{-1}=\left(\boldsymbol{S}^{-\mathbf{1}}+\beta \boldsymbol{H}\right)^{-1}
$$

where $H$ is defined from the second order derivative matrix $\boldsymbol{L}$ as $H=\boldsymbol{L}^{\mathbf{2}}$ (e.g, Quémerais et al. (2006); Koskinen et al. (2011)) and $\beta$ is analogous to a diffusion coefficient. The order of magnitude of the matrix terms in $L$ is around $(\Delta Z)^{-2}$, with $\Delta Z$ the vertical distance between two consecutive observations. Setting $\beta$ is a matter of tuning since there is no related physical process behind. We scale $\beta$ against the larger term of $\boldsymbol{S}^{\mathbf{- 1}}$ and we defined a free factor $\gamma$ so that we have $\beta=\gamma \Delta Z^{4} \times \max \left(\boldsymbol{S}_{\boldsymbol{i}, \boldsymbol{j}}^{\mathbf{- 1}}\right)$. We tested several values of $\gamma$ around 1 in order to remove small scale oscillations but keeping the variations comparable to the atmosphere scale height $(\simeq 40 \mathrm{~km})$. We found that $\gamma \simeq 0.316$ gives the requested effect for retrieval performed with VIMS data at wavelengths shorter than $3 \mu \mathrm{m}$.

To retrieve haze and methane, we proceed in two steps. We first use the methane windows where 
haze extinction alone acts on transmission. This allows to fix the haze vertical and spectral extinction. With this information about the haze layer, we then retrieve the vertical profile of methane mixing ratio. we also need to account for the spectral shift of the VIMS instrument (e.g. Maltagliati et al. (2015)) that we evaluated as part of the method. We use a subset of the data between 200 and $300 \mathrm{~km}$, in the middle atmosphere, and we seek for the best fit with different values of the shift. With a cost function $\left(\chi^{2}\right)$ computed in the methane bands only, we are able to draw the value of a $\chi^{2}$ as a function of the wavelength shift. We then evaluate the best value of the shift and the error bars. Wavelength shifts for VIMS channels giving the best matches of data are $2.64_{-0.21}^{+0.16} \mathrm{~nm}$ for $T 10$ and $9.40_{-0.09}^{+0.10} \mathrm{~nm}$ for $T 53$. This signicantly differs from $\mathrm{RC} 19^{1}$ prescriptions.

Appendix C Retrieval with wavelengths shorter than $2 \mu \mathrm{m}$ : We show here comparisons between our results and results from the previous analysis (Maltagliati et al. (2015)) obtained with the same dataset. The Figure 4 shows the retrieved vertical profiles of methane mixing ratio for the two observations $T 10$ and T53 as retrieved in this work and as retrieved by Maltagliati et al. (2015) in the bands at $1.4 \mu \mathrm{m}$ and $1.7 \mu \mathrm{m}$. We also display the retrieval that we can perform with the three bands at $1.2 \mu \mathrm{m}, 1.4 \mu \mathrm{m}$ and $1.7 \mu \mathrm{m}$ separately. This shows that results concerning the methane mixing ratio differ from the one made by Maltagliati et al. (2015), especially concerning the enriched layer at $165 \mathrm{~km}$ for $T 10$ which was not retrieved in the previous analysis. This feature is real since it appears separately in the three methane bands, in our analysis, with about the same contrast relative to the background. The background itself is also consistently found with the three different bands separately. The main difference between our work and the work by Maltagliati et al. (2015) is due to the high quality of the methane linelist that we use (e.g. Rey et al. (2018)). The corresponding fits of the data are shown in the Figure 5.

Appendix D Retrieval in the $2.3 \mu \mathrm{m}$ methane band: The Figure 6 shows the retrieved vertical profiles for the two observations $T 10$ and $T 53$, in the two wavelength intervals : between 2.0 and $2.8 \mu \mathrm{m}$ (band at $2.3 \mu \mathrm{m}$ ) and 0.88 to $2 \mu \mathrm{m}$. The modeled and retrieved spectra for the $2.3 \mu \mathrm{m}$ band are also shown. In our results, we immediately remark that the $2.3 \mu \mathrm{m}$ band can not allow for a safe retrieval of the methane abundance because another species strongly interacts with methane absorption. This clearly appears in the spectra that can not be match with the same quality of fit

\footnotetext{
${ }^{1}$ https://pds-imaging.jpl.nasa.gov/data/cassini/cassini_orbiter/vims-calibration-files/clark-et-al_vimsradiometric-calibration-pds-2018-v2.0.pdf
} 
than the spectra in between 0.88 to $2 \mu \mathrm{m}$ (Figure 1 of the article and Figure 5). Moreover, these poor fits are obtained with an excessive amount of methane. It was already well known that extra absorptions exist in the $\mathrm{C}-\mathrm{H}$ fundamental band at $3.4 \mu \mathrm{m}$ where methane does not absorb enough to explain observations (Bellucci et al. (2009); Maltagliati et al. (2015)). Here, we clearly see that there is also an undefined absorption in the $2.3 \mu \mathrm{m}$ band. As suggested by Maltagliati et al. (2015), ethane could be the absorbing gas, although it would appear surprising that a trace gas could compete with methane in one of its bands. If ethane is not responsible for the extra absorption in the $2.3 \mu \mathrm{m}$ band, it would mean that something is not yet understood in Titan's atmosphere opacity. For now, we consider that retrieval of methane with the $2.3 \mu \mathrm{m}$ band is not reliable and is only shown here for demonstration. 

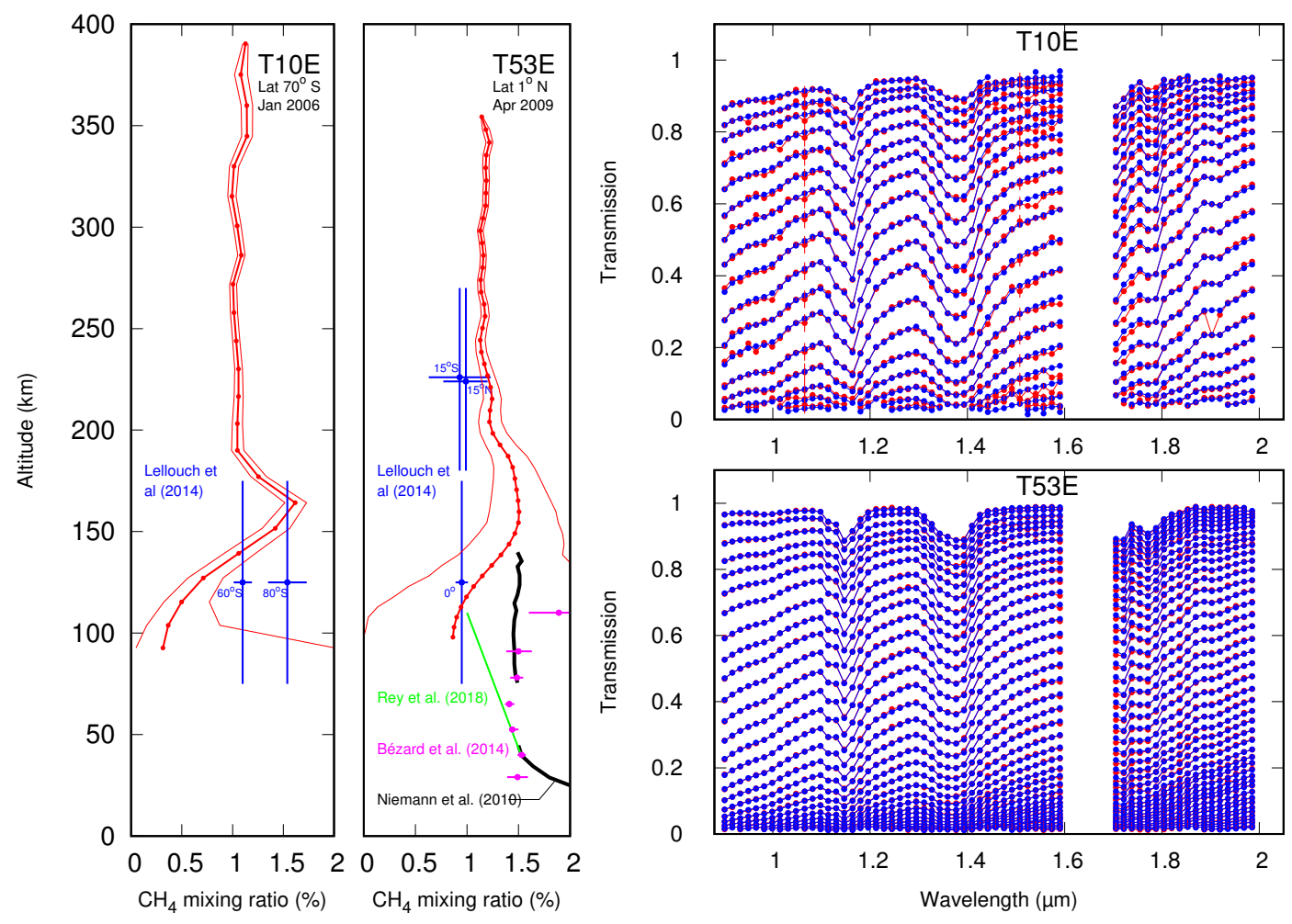

Figure 1: Two plots at left : Retrieved methane mixing ratio (red dots) and the related error envelope (red curves) with the two observation sets, between 0.88 and $2 \mu \mathrm{m}$. For the observation T10, we also plot the retrieval made with the CIRS observations at $125 \pm 50 \mathrm{~km}$ (Lellouch et al. (2014)) at $60^{\circ} \mathrm{S}$ in Jul. 2009 and at $80^{\circ} S$ in Nov 2007. For T53, we plot the methane mixing ratio retrieved with CIRS at $125 \pm 50$ $\mathrm{km}$ at equator in Dec. 2007, and at $225 \pm 50 \mathrm{~km}$ at $15^{\circ} \mathrm{N}$ in March 2007 and at $15^{\circ} \mathrm{S}$ in Sept 2006. We also show the methane mixing ratio retrieved by the Gas Chromatograph and Mass Spectrometer (GC-MS) (Niemann et al. (2010)) and with the Descent Imager Spectral Radiometer (DISR) (Bézard (2014); Rey et al. (2018)), both onboard Huygens which landed in January 2005 at $\simeq 10^{\circ} \mathrm{S}$. The spatial resolutions of our vertical profiles are $\pm 6.25 \mathrm{~km}$ and $\pm 2.5 \mathrm{~km}$ for $T 10$ and $T 53$ respectively. At right, comparison between observed transmissions (red dots with error bars) and the model retrieval (blue dots) in the spectral range selected to retrieve methane mixing ratio. The gap in spectra between 1.59 and $1.68 \mu \mathrm{m}$ is due to uncorrect spectels in VIMS instrument. 

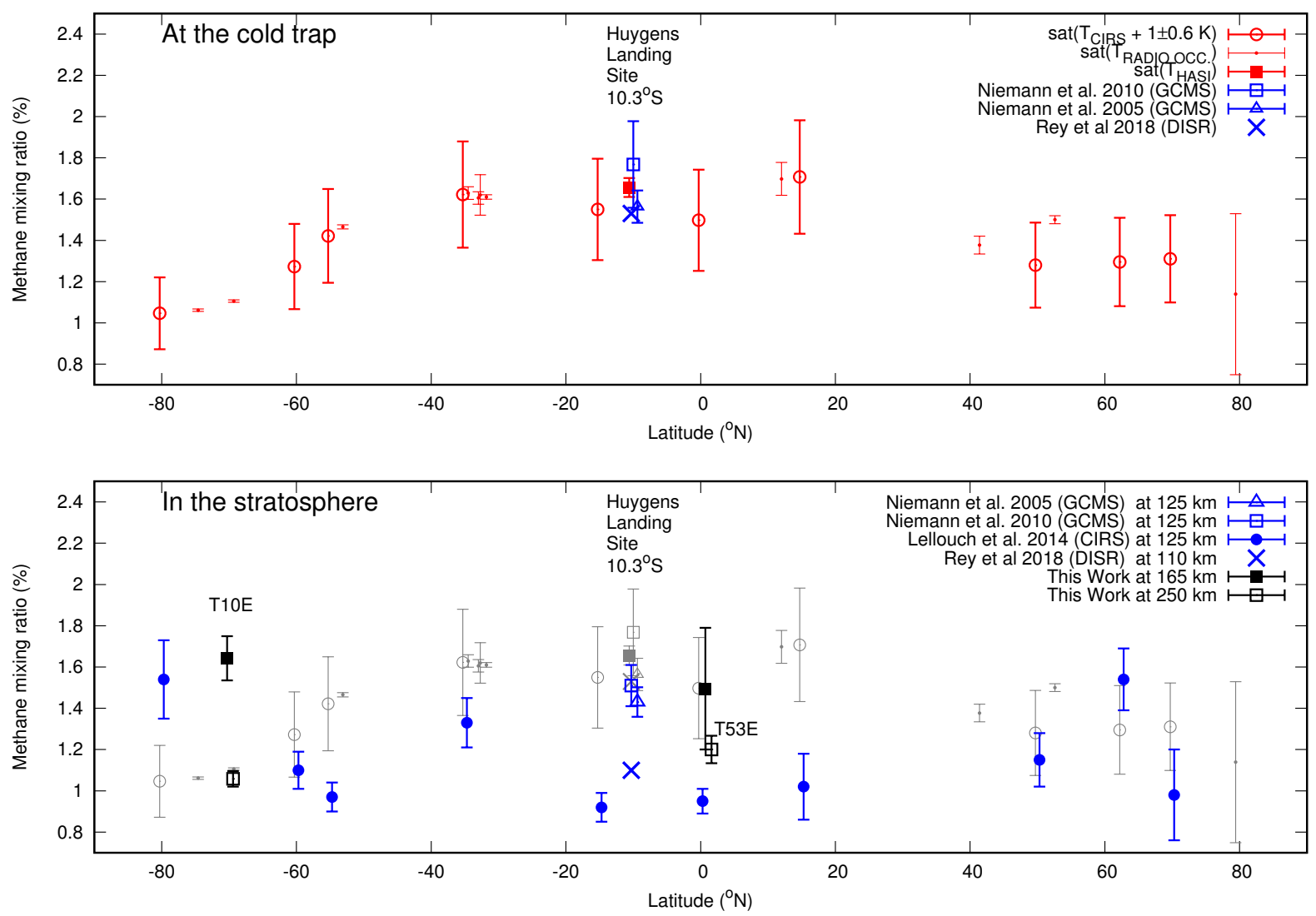

Figure 2: At top : Methane mixing ratio as a function of the latitude at the cold trap (altitude $\simeq 30$ km). We show values measured with the GC-MS (Niemann et al. (2005); Niemann et al. (2010)) and retrieved with the DISR (updated values by Rey et al. (2018)), both onboard Huygens. We also show saturation methane mixing ratio inferred from temperature measurements around the cold trap made by the Huygens Atmospheric Structure Instrument (HASI) (Fulchignoni et al. (2005)), from temperatures at $30 \pm 1 \mathrm{~km}$ retrieved with the Radio Occultation Experiment (Schinder et al. (2011, 2012, 2020)) and from temperatures retrieved by CIRS (Lellouch et al. (2014)) around the cold trap. We only selected dates before the North Spring Equinox. We add $+1 \pm 0.6 \mathrm{~K}$ to CIRS temperatures in order to account for a systematic bias in retrieved temperatures at the cold trap, compared to HASI and to the radio-occultation temperature retrievals (Lellouch et al. (2014)). This makes the saturation methane mixing ratios calculated from CIRS at the cold trap to be consistent with the 'in situ' measurements made by Huygens. At the cold trap, methane condensate is expected to be pure ice (Tokano et al. (2006)), then we use vapor pressures as published by Fray and Schmitt (2009). At bottom: Methane mixing ratios retrieved in the stratosphere for this study along with the retrieval made by CIRS at $125 \mathrm{~km}$ and DISR at $110 \mathrm{~km}$. The light grey symbols are those displayed in the top panel and reported here to facilitate comparisons. 


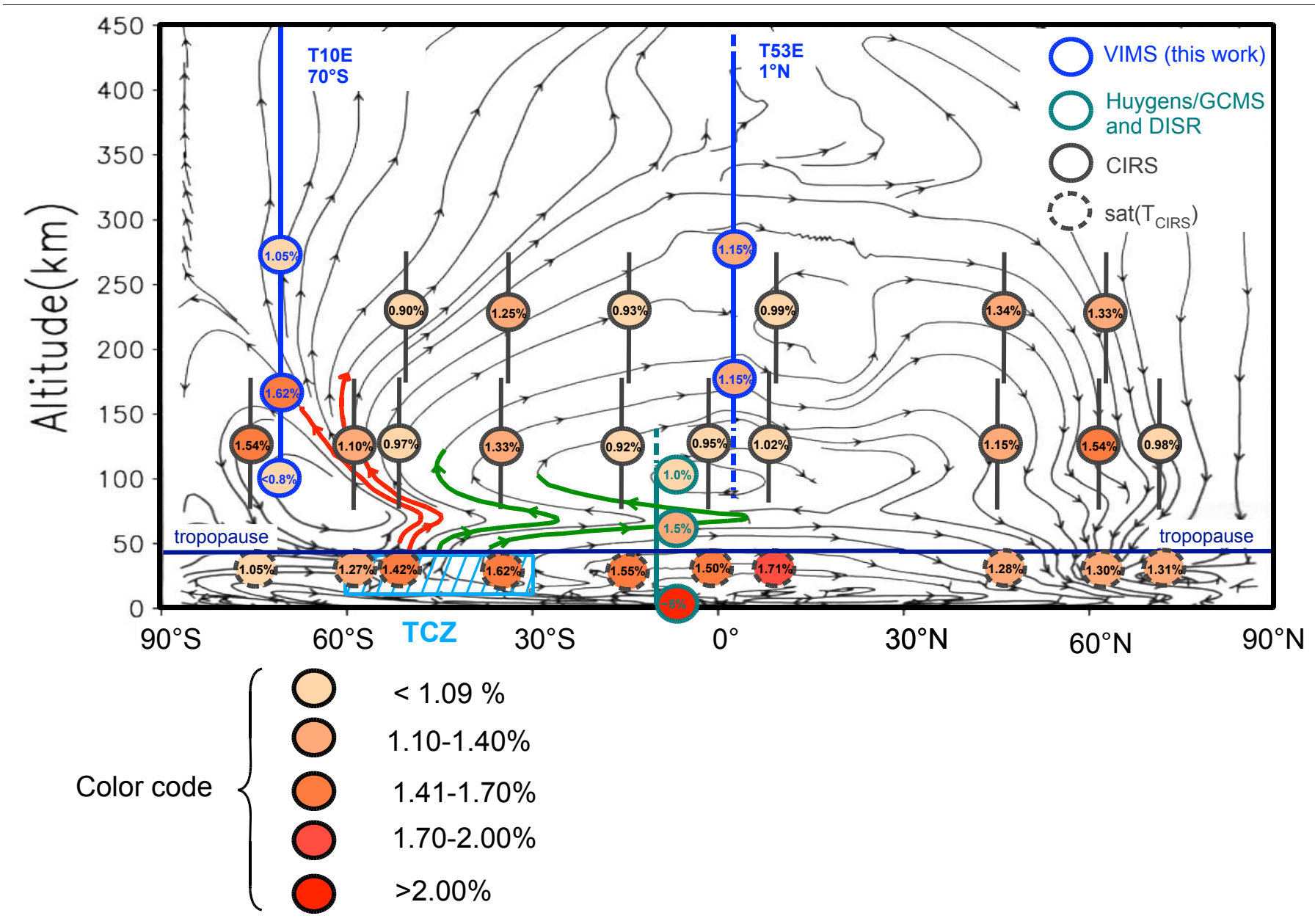

Figure 3: Circulation pattern averaged over one terrestrial year before the Northern Spring Equinox as predicted by the Titan IPSL-GCM (Rannou et al. (2006)) shown along with values of methane mixing ratios (values and color code) measured with Huygens (Niemann et al. (2010); Bézard (2014); Rey et al. (2018)), with CIRS (Lellouch et al. (2014)) and found in this work. Note, when comparing values, that CIRS values are averaged on broad vertical intervals of $\pm 50 \mathrm{~km}$ while other values are local (less than $\pm 6.3 \mathrm{~km}$ ). We also show the saturation mixing ratios for the cold trap temperature evaluated with CIRS (dashed circles). The mixing ratios shown here are reported, without their error bars, from Figure 2 except for the CIRS values at $225 \mathrm{~km}$. In this case, error bars are between $0.15 \%$ and $0.47 \%$. Two couples of wind streams are highlighted in red and green. They show how the methane-rich south tropical ascending branch at the $T C Z$, where convective clouds are frequently observed before equinox at latitude $45 \pm 15^{\circ} S$ (Cyan hatched box, Rodriguez et al. (2011); Turtle et al. (2018)), is connected to the methane rich layer at $70^{\circ} S$ and to the transition layer around $70 \mathrm{~km}$ in the equatorial region. High density of the stream lines is related to high mass flux. Therefore, the flux from the TCZ is quite constant and remains strong up to the edge of south polar region while the branch toward the equator rapidly diverges and weaken. It should be kept in mind that the circulation fluctuates with time around average patterns, as those shown here. 

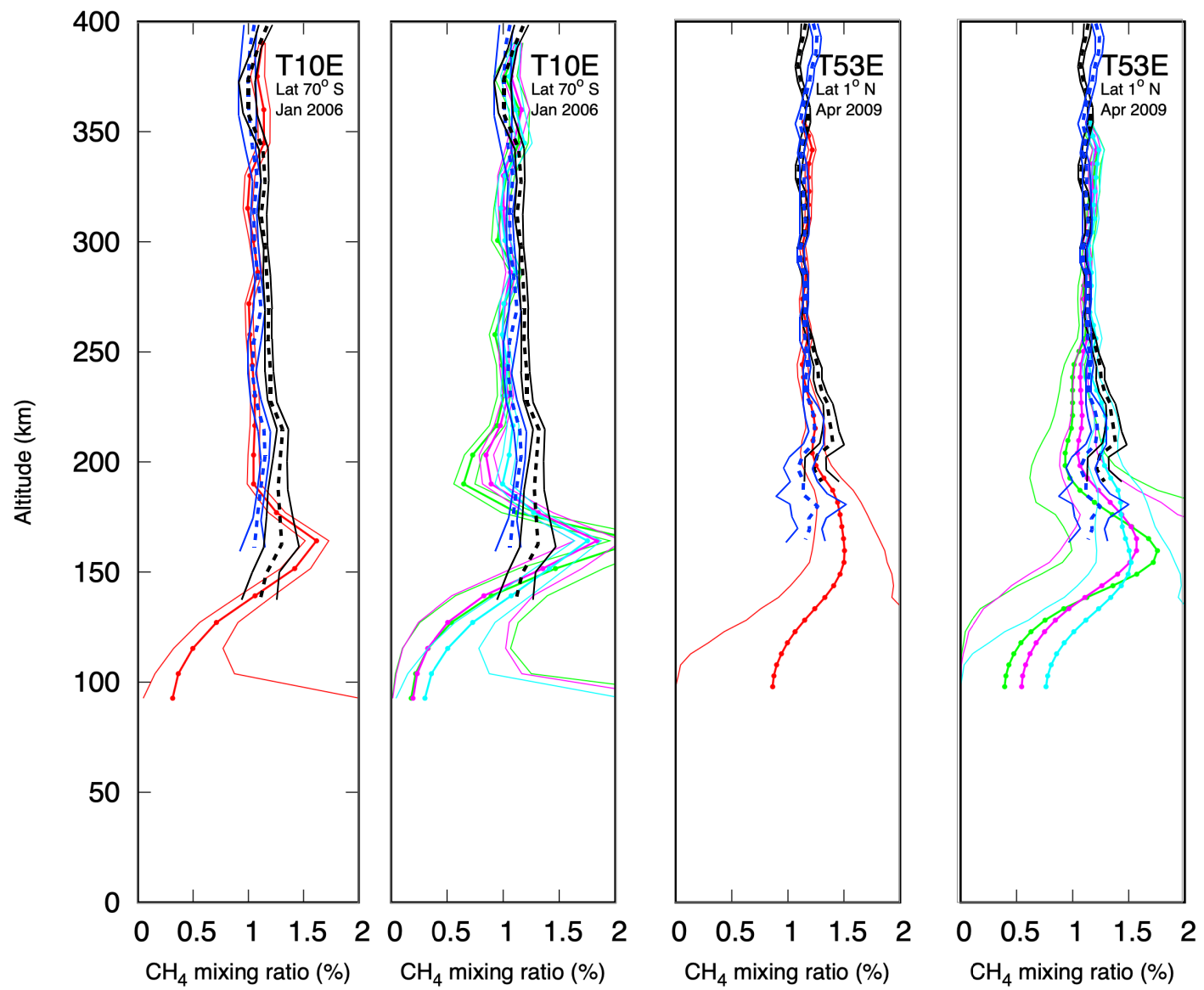

Figure 4: (First and third graphs from left) Methane mixing ratio (red dots) and the related error envelope (red curves) retrieved with the observation sets $T 10$ and $T 53$, and with data at wavelengths between 0.88 and $2.03 \mu \mathrm{m}$ shown along with the profiles retrieved by Maltagliati et al. (2015) with the $1.4 \mu \mathrm{m}$ (dash black) and the $1.7 \mu \mathrm{m}$ (dashed blue) methane bands. (Second and fourth graph from left) Methane mixing ratios (color dots) and the related error envelopes (color curves) obtained with the observation sets $T 10$ and $T 53$, and using the methane bands at $1.2 \mu \mathrm{m}$ (light green), $1.4 \mu \mathrm{m}$ (pink) and $1.7 \mu \mathrm{m}$ (cyan), shown along with the profiles retrieved by Maltagliati et al. (2015) with the $1.4 \mu \mathrm{m}$ (dash black) and the $1.7 \mu \mathrm{m}$ (dashed blue) methane bands. 


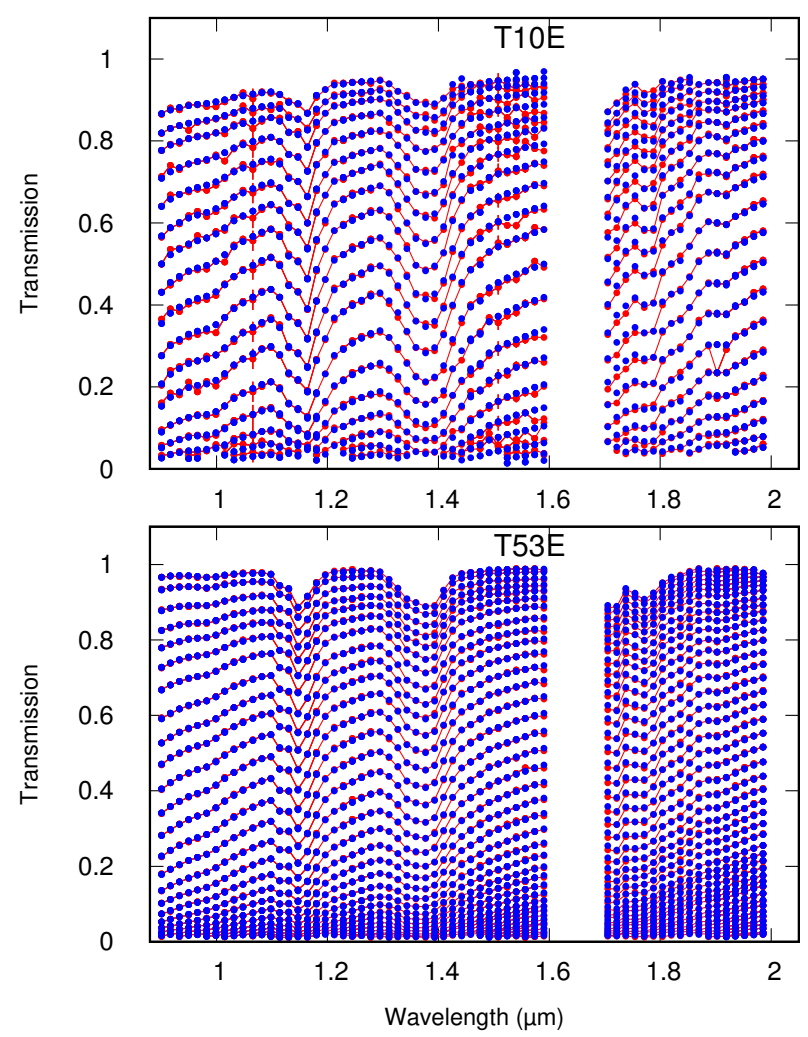

Figure 5: Comparison between observed transmissions (red dots with error bars) and the concatenation of the model retrieval (blue dots) made with the three methane bands separately. The gap in spectra between 1.59 and $1.68 \mu \mathrm{m}$ is due to uncorrect spectels in VIMS instrument. 

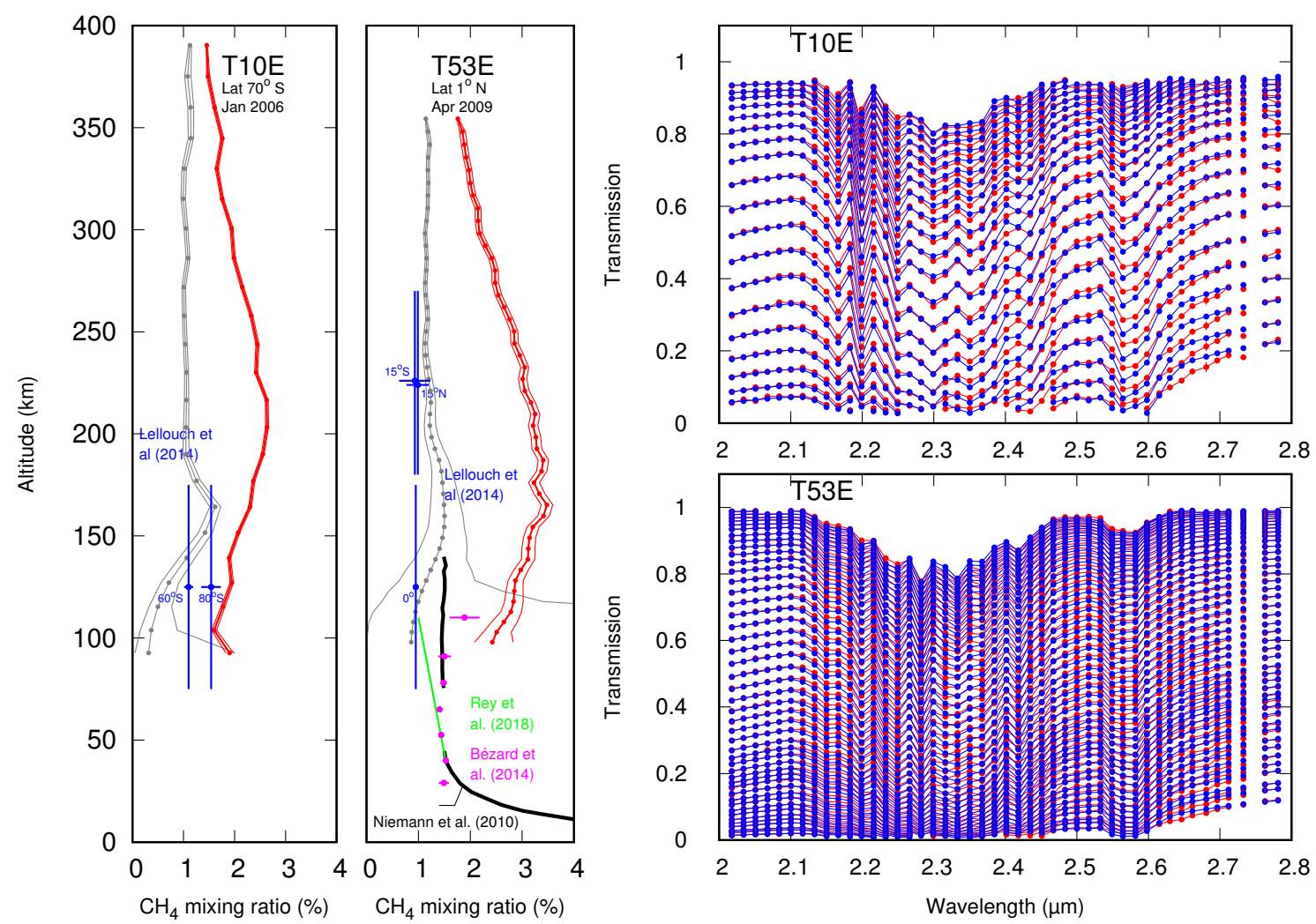

Figure 6: Two plots at left : Retrieved methane mixing ratio (red dots) and the related error envelope (red curves) with the observation sets $T 10$ and $T 53$, with wavelength between 2.03 and $2.8 \mu \mathrm{m}$. The grey profile are those plotted in the Figure 1 of the article, with retrievals performed with parts of the spectra between 0.88 and $2 \mu \mathrm{m}$. We also plot methane mixing ratios from other works (Lellouch et al. (2014), Niemann et al. (2010), Bézard (2014); Rey et al. (2018)). At right, comparison between observed transmissions (red dots with error bars) and the model retrieval (blue dots) in the spectral range selected to retrieve methane mixing ratio. The gap in spectra corresponds to wavelengths where ethane is known to absorb, but can not be modelled. 